\title{
Patient educational level and management of bipolar disorder
}

Alina Karanti, Lana Bublik, Mathias Kardell, Kristina Annerbrink, Paul Lichtenstein, Bo Runeson, Erik Pålsson and Mikael Landén

\section{Background}

Socioeconomic factors can affect healthcare management.

\section{Aims}

The aim was to investigate if patient educational attainment is associated with management of bipolar disorder.

\section{Method}

We included patients with bipolar disorder type $1(n=4289)$, type $2(n=4020)$ and not otherwise specified $(n=1756)$, from the Swedish National Quality Register for Bipolar Disorder (BipoläR). The association between patients' educational level and pharmacological and psychological interventions was analysed by binary logistic regression. We calculated odds ratios after adjusting for demographic and clinical variables.

\section{Results}

Higher education was associated with increased likelihood of receiving psychotherapy (adjusted odds ratio 1.34, 95\% Cl 91.22 1.46) and psychoeducation (adjusted odds ratio $1.18,95 \% \mathrm{Cl}$ 1.07-1.46), but with lower likelihood of receiving first-generation antipsychotics (adjusted odds ratio $0.76,95 \% \mathrm{Cl} 0.62-0.94$ ) and tricyclic antidepressants (adjusted odds ratio 0.76, $95 \% \mathrm{Cl}$ 0.59-0.97). Higher education was also associated with lower risk for compulsory in-patient care (adjusted odds ratio $0.79,95 \%$ Cl 0.67-0.93).

\section{Conclusions}

Pharmacological and psychological treatment of bipolar disorder differ depending on patients' educational attainment. The reasons for these disparities remain to be explained.

\section{Keywords}

Pharmacotherapy; psychotherapy; healthcare disparity; bipolar disorder; education.

\section{Copyright and usage}

(C) The Author(s), 2021. Published by Cambridge University Press on behalf of the Royal College of Psychiatrists. This is an Open Access article, distributed under the terms of the Creative Commons Attribution licence (http://creativecommons.org/ licenses/by/4.0/), which permits unrestricted re-use, distribution, and reproduction in any medium, provided the original work is properly cited.
Bipolar disorder is a serious psychiatric condition associated with high societal costs. ${ }^{1}$ Besides pharmacotherapy, adjunct psychoeducation and psychotherapy can help prevent relapse and increase treatment adherence.

\section{Socioeconomic factors and risk for bipolar disorder}

Socioeconomic factors, such as income, education and occupation, influence the risk for mental disorders. ${ }^{2}$ Interestingly, however, people with bipolar disorder more often belong to higher socioeconomic strata compared with controls or the general population. ${ }^{3}$ Likewise, a recent study suggested that higher socioeconomic status in parents was associated with increased risk of bipolar disorder in offspring. ${ }^{4}$ Educational attainment is a commonly used measure of socioeconomic status in epidemiological studies. ${ }^{5}$ It has high reliability and validity, ${ }^{5}$ is generally stable after early adulthood, and shapes future occupational opportunities and income potential. A Dutch study found that persons with bipolar disorder type 1 were more likely to have completed higher education than controls, ${ }^{6}$ and individuals with excellent school performance were shown to have a fourfold increased risk for developing bipolar disorder compared with those with average degrees. ${ }^{7}$ However, a Norwegian study found a similar level of education, but lower social status, in people with bipolar disorder compared with the general population. ${ }^{8}$

\section{Socioeconomic factors and management of bipolar disorder}

Besides being risk factors for disorders, socioeconomic factors might also be associated with the type of treatment patients receive. $^{9-12}$ Inequalities of treatment because of socioeconomic status, gender or race have generally received more attention in somatic care ${ }^{13,14}$ than in mental healthcare, and the literature is yet sparse regarding potential treatment inequalities in relation to educational level in psychiatry, let alone bipolar disorder.

The aim of this study was to investigate if educational level is associated with treatment and management of bipolar disorder in Sweden.

\section{Method}

\section{Study population}

We used data from the Swedish National Quality Register for Bipolar Disorder (BipoläR), which has been described previously. ${ }^{15}$ BipoläR contains data on bipolar diagnosis, including subtypes, comorbid psychiatric and somatic diagnoses, outcome data (such as number of depressive, hypomanic, manic and mixed episodes during the past 12 months; compulsory institutional care; and duration of in-patient care during the past 12 months), data on treatment (drug treatment, electroconvulsive therapy (ECT) and psychological treatment) and data on psychosocial functioning. BipoläR was launched in 2004, and currently, 240 psychiatric outpatient units report to the register. The participation is voluntary for both the physician and patient. All demographic areas across Sweden, and both public and private mental healthcare providers, are represented in BipoläR. Data is typically collected by the treating physician and entered into a web-based application. After the first registration, which can occur at any time point during out-patient treatment, information is updated annually.

We extracted data from 13304 unique individuals entered during the period 2004-2013. The reason for not including cases after 2013 is that BipoläR changed the registration form in 2014, whereupon the education variable was excluded. We included patients with bipolar disorder type 1, type 2 and not otherwise 
specified. We excluded individuals with schizoaffective disorder or other comorbid psychotic syndromes, to ensure that antipsychotics had been prescribed for bipolar disorder rather than for persistent psychotic symptoms. However, patients with bipolar diagnosis presenting psychotic symptoms restricted to affective episodes were included in our study population. We also excluded individuals with intellectual disabilities or autism spectrum disorders, considering that these conditions negatively affect educational level. Further, individuals with ongoing education and those younger than 22 years were excluded because they might not yet have reached their highest level of education. Finally, we excluded individuals with missing data on educational level or treatment. We analysed the remaining 10065 individuals with bipolar disorder, of which 4289 were diagnosed with bipolar disorder type 1, 4020 were diagnosed with bipolar disorder type 2 and 1756 were diagnosed with bipolar disorder not otherwise specified.

\section{Exposure}

The main exposure was level of education. The highest completed education level for each patient in the register was reported as follows: 'not completed primary school', 'completed primary school', 'completed upper secondary school' or 'at least 2 years post-secondary education'. In Sweden, primary school is 9 years and has been mandatory since 1962 . Three years of upper secondary school education is optional, but presently very common and required for many occupations. By 2014, almost half of Sweden's inhabitants aged 25-64 years had upper secondary education, more than a third had some form of post-secondary education and more than a quarter had at least 3 years of university education. ${ }^{16}$ In our analyses, we therefore dichotomised the education variable into 'no university studies' and 'university studies'.

\section{Outcomes}

Pharmacological treatment variables were current treatment with mood stabilisers as a group, lithium, lamotrigine, divalproex, antipsychotics as a group, quetiapine/aripiprazole/olanzapine as a group, first-generation antipsychotics (fluphenazine, flupenthixol, haloperidol, chlorpromazine, chlorprothixene, perphenazine, prochlorperazine, sulpiride, thioridazine and zuclopenthixol), antidepressants as a group, tricyclic antidepressants (amitriptyline, imipramine, clomipramine, nortriptyline, trimipramine, lofepramine and maprotiline) and benzodiazepines. Other treatment variables were having received ECT at any time, having had at least ten sessions of psychological treatment, having received psychoeducation for bipolar disorder, having had psychoeducation provided to next of kin, compulsory in-patient care and duration of in-patient care during the past 12 months. All outcome variables except duration of in-patient care were dichotomous, with the answer 'yes' coded as 1 .

\section{Covariates}

Demographic variables, such as age and psychosocial functioning (captured with the Global Assessment of Functioning (GAF) scale, according to the DSM-IV ${ }^{17}$ ), were included as covariates. We included additional covariates in relation to specific outcome variables. For lithium and lamotrigine, we adjusted for bipolar subtype because lithium is more likely to be used in type 1 and lamotrigine is more likely to be used in type 2. For antipsychotic treatment, we included the number of elated and mixed episodes during the past 12 months as a confounder, because manic/mixed episodes are commonly treated with antipsychotics. For antidepressant drug treatment and ECT, we included the number of depressive episodes during the past 12 months as a covariate. For benzodiazepine treatment, we added comorbid anxiety disorders as a covariate. For psychological treatment, we included comorbid personality disorders as a covariate because it is an indication for psychotherapy. All covariates except age, GAF function score and number of affective episodes were dichotomous, with the answer 'yes' coded as 1 .

\section{Statistical analyses}

SPSS version 22 (IBM SPSS Statistics 22.0) for Windows was used for statistical analyses. Associations between educational level and interventions were analysed with binary logistic regression. Adjusted odds ratios were computed for each outcome variable by performing two regression models. In the first regression model, we adjusted for age and GAF function score. In the second regression model, we adjusted for additional confounders as described above.

We performed two sensitivity analyses. In the first sensitivity analysis, we excluded all individuals under 26 years old, to minimise the risk for ongoing education that might change educational status. The results remained identical with our main analysis (data not shown). In the second sensitivity analysis, we stratified the study population into three groups, according to age at entering the BipoläR register (age groups 22-44, 45-64 and >64 years), because there could be an age effect with respect to educational level across generations.

\section{Ethics and consent statement}

The study was approved by the regional Ethical Review Board in Gothenburg (approval number 294-11), and all procedures contributing to this work comply with the ethical standards of the relevant national and institutional committees on human experimentation and with the Helsinki Declaration of 1975, as revised in 2008. According to Swedish law, inclusion in Swedish quality registers follows an opt-out procedure, where patients are informed and may decline to participate, in which case data cannot be recorded. All analyses were conducted on a de-identified data-set, where neither individual patients nor physicians can be identified or traced in the material.

\section{Results}

\section{Study sample and characteristics}

Clinical and demographical data for the entire study sample of 10065 patients are shown in Table 1.

\section{Education and interventions}

The educational level was not associated with treatment with mood stabilisers as a group, lithium, lamotrigine or valproic acid (Table 2). ${ }^{18}$ Nor were there any associations between educational level and antipsychotics as a group, or the group of quetiapine/aripiprazole/olanzapine, after adjusting for the number of elated or mixed episodes. However, the use of first-generation antipsychotics was higher in the low-education group. There was no association between the level of education and ECT or antidepressants as a group, after adjusting for the number of depressive episodes. However, tricyclic antidepressants were more common among individuals with lower education than among those with higher education. Further, there was no association between benzodiazepines and educational level after adjusting for comorbid anxiety disorders. However, higher level of education was associated with increased likelihood of having received psychoeducation, psychoeducation 
Table 1 Clinical and demographical characteristics of 10065 patients with bipolar disorder

\begin{tabular}{|lccc|}
\hline & Not & & \\
& university & University & $P$-value \\
\hline Bipolar subtype & & & $<0.001$ \\
Type 1, \% (n) & $42.8(2628)$ & $42.4(1661)$ & \\
Type 2, \% (n) & $38.6(2373)$ & $42.0(1647)$ & \\
Not otherwise specified, \% (n) & $18.6(1143)$ & $15.6(613)$ & \\
Female, \% (n) & $62.1(3814)$ & $60.9(2389)$ & 0.248 \\
Age, mean (s.d.) & $48.0(15.5)$ & $49.8(13.9)$ & $<0.001$ \\
Global Assessment of Functioning & $61.5(19.0)$ & $66.9(17.5)$ & $<0.001$ \\
function score, mean (s.d.) & & & \\
Number of medications, mean & $2.4(1.4)$ & $2.2(1.3)$ & $<0.001$ \\
Comorbidities, \% (n) & & & \\
Substance misuse & $5.4(323)$ & $4.0(151)$ & 0.001 \\
Anxiety disorders & $13.5(803)$ & $9.9(376)$ & $<0.001$ \\
Personality disorders & $4.2(248)$ & $2.4(93)$ & $<0.001$ \\
Interventions, \% (n) & & & \\
Mood stabilisers as a group & $85.1(5226)$ & $85.1(3337)$ & 0.948 \\
$\quad$ Lithium & $56.2(3451)$ & $57.6(2259)$ & 0.154 \\
Lamotrigine & $25.7(1582)$ & $25.7(1006)$ & 0.918 \\
Divalproex & $10.7(655)$ & $9.5(373)$ & 0.064 \\
Antipsychotics as a group & $35.5(2179)$ & $31.3(1227)$ & $<0.001$ \\
Quetiapine/aripiprazole/ & $25.1(1540)$ & $23.5(923)$ & 0.083 \\
olanzapine & & & \\
First-generation antipsychotics & $4.8(297)$ & $3.4(134)$ & 0.001 \\
Antidepressants as a group & $45.0(2764)$ & $44.6(1747)$ & 0.671 \\
Tricyclic antidepressants & $3.2(196)$ & $2.3(92)$ & 0.008 \\
Benzodiazepines & $41.7(1531)$ & $39.1(933)$ & 0.043 \\
Electroconvulsive therapy & $21.9(1308)$ & $21.0(803)$ & 0.143 \\
Psychotherapy & $65.2(4003)$ & $69.0(2705)$ & $<0.001$ \\
Psychoeducation & $21.9(1347)$ & $24.7(968)$ & 0.001 \\
Psychoeducation to next of kin & $14.6(900)$ & $17.3(677)$ & $<0.001$ \\
Compulsory in-patient care & $13.9(475)$ & $10.3(245)$ & $<0.001$ \\
Duration of in-patient care, mean & $34.7(40.3)$ & $33.3(38.3)$ & 0.632 \\
(s.d.), days & & & \\
\hline & & & \\
\hline
\end{tabular}

for next of kin and psychotherapy. Finally, individuals with lower education had a higher rate of compulsive in-patient care.

In the sensitivity analysis stratified by age, the results were in concordance with main analysis despite some results falling short of statistical significance, which is likely because of lower statistical power (see Supplementary Table 1 available at https://doi.org/10. 1192/bjo.2021.19).

\section{Discussion}

We investigated if patients' level of education was associated with the management of bipolar disorder in a total cohort of 10056 individuals. We found that higher education was associated with increased likelihood of receiving psychological treatment and psychoeducation, and decreased likelihood of treatment with first-generation antipsychotics and tricyclic antidepressants. ${ }^{18}$ Finally, compulsory in-patient care was more common in the low-education group. ${ }^{18}$

There are few previous studies on the importance of patients' education for bipolar disorder management. Levine et $\mathrm{al}^{19} \mathrm{did}$ not find any association between educational level and pharmacological treatment in a USA study of 457 patients with bipolar disorder type 1 , who listed their medication use within the past month. Park et $\mathrm{al}^{20}$ found a negative correlation between higher education (college degree) and treatment with second-generation antipsychotics in bipolar disorder type 1 . Not only were these studies several order of magnitudes smaller than ours, but the diverging findings could also be a result of differing healthcare organisation and inequality indices across countries. This is exemplified by a study from the USA, where higher education (college) predicted benzodiazepine use in 482 patients with bipolar disorder type 1 or $2 .{ }^{21}$ The
Table 2 Association between educational level and interventions for bipolar disorder

\begin{tabular}{|c|c|c|}
\hline Intervention & $\begin{array}{c}\text { Model } 1^{\mathrm{a}} \\
\mathrm{aOR}^{\mathrm{b}}(95 \% \mathrm{Cl})\end{array}$ & $\begin{array}{c}\text { Model } 2 \\
\operatorname{aOR}^{\mathrm{b}}(95 \% \mathrm{Cl})\end{array}$ \\
\hline Mood stabilisers as a group & $0.96(0.86-1.07)$ & \\
\hline Lithium & $0.97(0.89-1.05)$ & $0.99^{\mathrm{C}}(0.91-1.08)$ \\
\hline Lamotrigine & $1.11(1.01-1.22)$ & $1.08^{\mathrm{C}}(0.98-1.19)$ \\
\hline Divalproex & $0.92(0.81-1.06)$ & \\
\hline Antipsychotics as a group & $0.90(0.83-0.99)$ & $0.94^{d}(0.86-1.03)$ \\
\hline $\begin{array}{l}\text { Quetiapine/aripiprazole/ } \\
\text { olanzapine }\end{array}$ & $1.01(0.92-1.11)$ & $1.04^{\mathrm{d}}(0.94-1.14)$ \\
\hline First-generation antipsychotics & $0.71(0.58-0.88)$ & $0.76^{\mathrm{d}}(0.62-0.94)$ \\
\hline Antidepressants as a group & $1.05(0.96-1.14)$ & $1.07^{\mathrm{e}}(0.98-1.17)$ \\
\hline Tricyclic antidepressants & $0.74(0.58-0.96)$ & $0.76^{\mathrm{e}}(0.59-0.97)$ \\
\hline Benzodiazepines & $0.97(0.87-1.09)$ & $0.99^{f}(0.88-1.10)$ \\
\hline Electroconvulsive therapy & $0.94(0.85-1.04)$ & $0.94^{\mathrm{e}}(0.85-1.04)$ \\
\hline Psychotherapy & $1.31(1.20-1.43)$ & $1.34^{g}(1.22-1.46)$ \\
\hline Psychoeducation & $1.18(1.07-1.30)$ & \\
\hline Psychoeducation for next of kin & $1.24(1.11-1.38)$ & \\
\hline Compulsory in-patient care & $0.79(0.67-0.93)$ & \\
\hline \multicolumn{3}{|c|}{$\begin{array}{l}\text { a. Model } 1 \text { was adjusted for age and Global Assessment of Functioning function score. } \\
\text { b. Adjusted odds ratio (aOR) for educational level versus intervention. An aOR }>1 \text { means } \\
\text { that the intervention is more common in the group with higher education. } \\
\text { c. Model 2, adjusted for bipolar type. } \\
\text { d. Model 2, adjusted for number of manic, hypomanic and mixed episodes. } \\
\text { e. Model 2, adjusted for number of depressive episodes. } \\
\text { f. Model 2, adjusted for comorbid anxiety disorders. } \\
\text { g. Model 2, adjusted for comorbid personality disorders. }\end{array}$} \\
\hline
\end{tabular}

authors explained this by limited coverage of benzodiazepines by some state insurance programmes. In Sweden, all citizens are covered by the same insurance programme, and we could not replicate the USA study finding. Regarding psychological interventions, three previous studies, although not limited to bipolar disorder, are in line with our findings that higher educational level is associated with higher probability of receiving psychotherapy. ${ }^{22-24}$

It might not be possible to fully explain the mechanisms driving inequality because research on healthcare disparities simultaneously study differences in access, need and demand, without always being able to adjust for one or the other. With this caveat, dissimilarities may arise on the system, clinician or patient level. ${ }^{18}$

From a systems perspective, Sweden is a welfare state, with relatively low health inequality and equal access to healthcare. Yet, inequalities in mental health and healthcare have been reported in Sweden. ${ }^{25,26}$ Psychiatric units in socioeconomically disadvantaged geographical areas may lack access to psychotherapists and have reduced possibility to offer the treatment, regardless of patients' educational level. ${ }^{18}$

As to the role of clinicians, we are not aware of any studies suggesting that patients with higher education have a greater need for, or would respond better to, psychological treatments. Conversely, we are not aware of any studies suggesting that patients with lower education would respond better to first-generation antipsychotics or tricyclic antidepressants. Interestingly, it has been suggested that the patient and doctor being on the same socioeconomic level could influence a doctor's choice for drug prescription. $^{27}$

With respect to the role of patients, expectations and ability to demand a specific treatment have been shown to be related to the patient's educational level, and might drive differences in psychological treatments. ${ }^{9}$ Our finding that higher-educated patients with bipolar disorder received fewer first-generation antipsychotics and tricyclic antidepressants could also be 'patient-mediated', as persons with higher education may have better access to drug information, including side-effects. ${ }^{18}$ Our finding of an inverse relationship between use of first-generation antipsychotics and educational level has also been demonstrated in a recent study in elderly patients 
with and without dementia in Sweden. ${ }^{11}$ Finally, educational level might be associated with the severity or type of illness, which in turn, warrants different interventions. For example, higher educational level is a proxy for cognitive reserve, which has been suggested to be associated with course and functional outcome of bipolar disorder. ${ }^{28}$ Although our regression models were adjusted for GAF function score, number of mood episodes and comorbid personality disorder, we could not control for differences in cognitive function or the severity of the disorder. ${ }^{18}$

It is worth considering that the drug treatment disparities we found might be attributable to differences in the income rather than educational level per se, as educational level reflects socioeconomic position. Swedish public services do not meet the need and demand for psychological treatment, and patients with higher education might have the financial means to afford private psychotherapy. ${ }^{18}$ Unfortunately, we cannot tell if psychotherapy was provided by private or public providers. Psychoeducation is, however, provided by publicly funded psychiatric units in Sweden, and the difference we found is unlikely to be explained by income differences. With respect to drug treatment, new drugs tend to be more expensive, and have been shown to be prescribed more frequently in higher socioeconomic groups. ${ }^{10}$ However, income differences across educational groups are less likely to explain our findings, not only because socioeconomic differences in Sweden are modest from an international perspective, but also because drug treatment is highly subsidised: after an initial cost of 2300 Swedish kronor (approximately US $\$ 240$ as of February 2019), patients are eligible for free prescribed drugs for the remainder of a 12 -month period, through the social welfare system. ${ }^{18}$ This suggests that the disparities in prescription drugs we find are likely to be associated with educational level rather than income. ${ }^{18}$ In support of this notion, Nordin et $\mathrm{al}^{12}$ found a positive relationship between education and drug utilisation, after controlling for income.

\section{Strengths and limitations}

We studied a large, representative real-world clinical cohort of Swedish patients with bipolar disorder. Among limitations that need to be considered is the cross-sectional study design, which does not allow causal inference. We further lacked information on the indication for drug prescriptions (e.g. mood stabilisers, antipsychotic medication). To partially address this, we excluded individuals with psychotic conditions, including schizoaffective disorder, and we also adjusted for bipolar subtype, comorbid psychiatric conditions and number of depressive or elated episodes. Third, although registry-based bipolar diagnoses in Sweden have been shown to have good validity overall, ${ }^{29}$ and the BipoläR diagnoses are made according to the DSM-IV, diagnoses were made in clinical routine and not according to a research protocol. On the other hand, this procedure reflects real-world praxis. Finally, BipoläR do not include all patients with bipolar diagnosis in Sweden; however, BipoläR has previously been found to be representative to the whole Swedish bipolar disorder population, ${ }^{30}$ and the gender distribution and education levels in our study sample are similar to the general bipolar disorder population in Sweden and to other large bipolar disorder study samples. ${ }^{16,31}$

In conclusion, we find that the level of education is associated with differences in bipolar disorder management in Sweden. ${ }^{18} \mathrm{We}$ fail to identify any medical rationale behind these differences, which thus may indicate unequal treatment. ${ }^{18}$ To disentangle the mechanism underlying these disparities, studies would need to include data on specific indications for pharmacological treatments, geographical differences in access to care, and patients' and clinicians' attitudes. ${ }^{18}$
Alina Karanti (D) PhD, Department of Psychiatry and Neurochemistry, Institute of Neuroscience and Physiology, Sahlgrenska Academy, University of Gothenburg, Sweden; Lana Bublik, MD, Department of Psychiatry and Neurochemistry, Institute of Neuroscience and Physiology, Sahlgrenska Academy, University of Gothenburg, Sweden; Mathias Kardell, Department of Psychiatry and Neurochemistry, Institute of Neuroscience and Physiology, Sahlgrenska Academy, University of Gothenburg, Sweden; Kristina Annerbrink $M D, P D$, Department of Psychiatry and Neurochemistry, Institute of Neuroscience and Physiology, Sahlgrenska Academy, University of Gothenburg, Sweden; Paul Lichtenstein, PhD, Department of Medical Epidemiology and Sweden; Paul Lichtenstein, PhD, Department of Medical Epidemiology and
Biostatistics, Karolinska Institutet, Sweden; Bo Runeson, MD, PhD, Department of Clinical Neuroscience, Centre for Psychiatry Research, Karolinska Institutet, Sweden; Erik Pålsson, PhD, Department of Psychiatry and Neurochemistry, Institute of Neuroscience and Physiology, Sahlgrenska Academy, University of Gothenburg, Sweden; Mikael Landén, MD, PhD, Department of Medical Epidemiology and Biostatistics, Karolinska Institutet, Sweden; and Department of Psychiatry and Neurochemistry, Institute of Neuroscience and Physiology, Sahlgrenska Academy, University of Institute of Neuroscience
Gothenburg, Sweden

Correspondence: Alina Karanti. Email: alina.karanti@vgregion.se

First received 29 Dec 2019, final revision 22 Jan 2021, accepted 2 Feb 2021

\section{Supplementary material}

Supplementary material is available online at https://doi.org/10.1192/bjo.2021.19.

\section{Data availability}

The data-sets generated and/or analysed during the current study are not publicly available because of the Swedish law for register data, but are available from the corresponding author, A.K., on reasonable request.

\section{Acknowledgements}

We wish to thank the Swedish National Quality Register for Bipolar Disorders (BipoläR)

\section{Author contributions}

A.K., L.B., K.A., E.P. and M.L. analysed and interpreted the data, and contributed to writing the manuscript. M.K. performed the statistical analysis. B.R. and P.L. contributed to the interpretation of the results. All authors read and approved the final manuscript.

\section{Funding}

This research was supported by grants from the Swedish Research Council (2018-02653) and the swedish Federal Government under the LUA/ALF agreement (ALFGBG-716801).

\section{Declaration of interest}

A.K., L.B., M.K., K.A., E.P., P.L. and B.R. declare that they have no competing interests. M.L. declares receiving lecture honoraria from Lundbeck Pharmaceutical over the past 36 months.

\section{References}

1 Ekman M, Granstrom O, Omerov S, Jacob J, Landen M. The societal cost of bipolar disorder in Sweden. Soc Psychiatry Psychiatr Epidemiol 2013; 48(10): 1601-10.

2 Lorant V, Deliege D, Eaton W, Robert A, Philippot P, Ansseau M. Socioeconomic inequalities in depression: a meta-analysis. Am J Epidemiol 2003; 157(2): 98-112.

3 Goodwin FK, Jamison KR. Manic-Depressive Illness: Bipolar Disorders and Recurrent Depression. Oxford University Press, 2007.

4 Tsuchiya KJ, Agerbo E, Byrne M, Mortensen PB. Higher socio-economic status of parents may increase risk for bipolar disorder in the offspring. Psychol Med 2004; 34(5): 787-93.

5 Liberatos $\mathrm{P}$, Link BG, Kelsey JL. The measurement of social class in epidemiology. Epidemiol Rev 1988; 10(1): 87-121.

6 Vreeker A, Boks MP, Abramovic L, Verkooijen S, van Bergen AH, Hillegers MH, et al. High educational performance is a distinctive feature of bipolar disorder: a study on cognition in bipolar disorder, schizophrenia patients, relatives and controls. Psychol Med 2016; 46(4): 807-18.

7 MacCabe JH, Lambe MP, Cnattingius S, Sham PC, David AS, Reichenberg A et al. Excellent school performance at age 16 and risk of adult bipolar disorder: national cohort study. Br J Psychiatry 2010; 196(2): 109-15.

8 Schoeyen $\mathrm{HK}$, Birkenaes $\mathrm{AB}$, Vaaler $\mathrm{AE}$, Auestad BH, Malt UF, Andreassen OA, et al. Bipolar disorder patients have similar levels of education but lower socioeconomic status than the general population. J Affect Disord 2011; 129(1-3): 68-74. 
9 Weitoft GR, Rosen M, Ericsson O, Ljung R. Education and drug use in Sweden-a nationwide register-based study. Pharmacoepidemiol Drug Saf 2008; 17(10): 1020-8.

10 Haider SI, Johnell K, Ringback Weitoft G, Thorslund M, Fastbom J. Patient educational level and use of newly marketed drugs: a register-based study of over 600,000 older people. Eur J Clin Pharmacol 2008; 64(12): 1215-22.

11 Wastesson JW, Ringback Weitoft G, Johnell K. Educational disparities in antipsychotic drug use among older people with and without dementia in Sweden. Acta Psychiatr Scand 2015; 132(1): 20-8.

12 Nordin M, Dackehag M, Gerdtham UG. Socioeconomic inequalities in drug utilization for Sweden: evidence from linked survey and register data. Soc Sci Med 2013; 77: 106-17

13 Wastesson JW, Weitoft GR, Parker MG, Johnell K. Educational level and use of osteoporosis drugs in elderly men and women: a Swedish nationwide register-based study. Osteoporos Int 2013; 24(2): 433-42.

14 Dzayee DA, Moradi T, Beiki O, Alfredsson L, Ljung R. Recommended drug use after acute myocardial infarction by migration status and education level. Eur J Clin Pharmacol 2015; 71(4): 499-505.

15 Karanti A, Bobeck C, Osterman M, Kardell M, Tidemalm D, Runeson B, et al. Gender differences in the treatment of patients with bipolar disorder: a study of 7354 patients. J Affect Disord 2015; 174: 303-9.

16 Statistics Sweden. Yearbook of Educational Statistics 2015. Statistical agency and producer, 2015 (http://www.scb.se/uf0524-en).

17 American Psychiatric Association. Diagnostic and Statistical Manual of Mental Disorders (4th ed., text revision). American Psychiatric Association, 2000.

18 Karanti A. Bipolar Disorders: Subtypes, Treatments, and Health Inequalities. University of Gothenburg, 2020 (http://hdl.handle.net/2077/62687).

19 Levine J, Chengappa KN, Brar JS, Gershon S, Yablonsky E, Stapf D, et al. Psychotropic drug prescription patterns among patients with bipolar I disorder. Bipolar Disord 2000; 2(2): 120-30.

20 Park DY, Goffin KC, Shah S, Yuen LD, Holtzman JN, Hooshmand F, et al Differential prevalence and demographic and clinical correlates of secondgeneration antipsychotic use in bipolar I versus bipolar II disorder. J Psychiatr Res 2016; 76: 52-8.

21 Bobo WV, Reilly-Harrington NA, Ketter TA, Brody BD, Kinrys G, Kemp DE, et al. Complexity of illness and adjunctive benzodiazepine use in outpatients with bipolar I or II disorder: results from the Bipolar CHOICE study. I Clin Psychopharmacol 2015; 35(1): 68-74.

22 Briffault X, Sapinho D, Villamaux $M$, Kovess V. Factors associated with use of psychotherapy. Soc Psychiatry Psychiatr Epidemiol 2008; 43(2): 165-71.

23 Suoyrjo H, Hinkka K, Kivimaki M, Klaukka T, Pentti J, Vahtera J. Allocation of rehabilitation measures provided by the Social Insurance Institution in Finland: a register linkage study. J Rehabil Med 2007; 39(3): 198-204.

24 Jokela M, Batty GD, Vahtera J, Elovainio M, Kivimaki M. Socioeconomic inequalities in common mental disorders and psychotherapy treatment in the UK between 1991 and 2009. Br J Psychiatry 2013; 202: 115-20.

25 Löfqvist T, Burström B, Walander A, Ljung R. Inequalities in avoidable hospitalisation by area income and the role of individual characteristics: a population-based register study in Stockholm County, Sweden. BMJ Qual Saf 2014; 23(3): 206-14.

26 Hiyoshi A, Kondo N, Rostila M. Increasing income-based inequality in suicide mortality among working-age women and men, Sweden, 1990-2007: is there a point of trend change? J Epidemiol Community Health 2018; 72(11): 1009-15.

27 Van Ryn M, Burke J. The effect of patient race and socio-economic status on physicians' perceptions of patients. Soc Sci Med 2000; 50(6): 813-28.

28 Anaya C, Torrent C, Caballero FF, Vieta E, Bonnin C, Ayuso-Mateos JL, et al. Cognitive reserve in bipolar disorder: relation to cognition, psychosocial functioning and quality of life. Acta Psychiatr Scand 2016; 133(5): 386-98.

29 Sellgren $\mathrm{C}$, Landén $\mathrm{M}$, Lichtenstein $\mathrm{P}$, Hultman $\mathrm{CM}$, Långström N. Validity of bipolar disorder hospital discharge diagnoses: file review and multiple register linkage in Sweden. Acta Psychiatr Scand 2011; 124(6): 447-53.

30 Pålsson E, Landén M. The Swedish National Quality Register for Bipolar Disorder - BipoläR. Annual Report 2017. Västra Götalandsregionen, 2018 (https://registercentrum.blob.core.windows.net/bipolar/r/Bipolar_arsrapport_-2017SJevIXQEDQ.pdf).

31 Kogan JN, Otto MW, Bauer MS, Dennehy EB, Miklowitz DJ, Zhang HW, et al. Demographic and diagnostic characteristics of the first 1000 patients enrolled in the Systematic Treatment Enhancement Program for Bipolar Disorder (STEPBD). Bipolar Disord 2004; 6(6): 460-9.
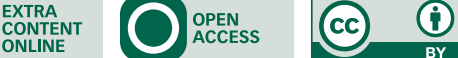